\title{
Re-evaluation of early Cambrian ocean oxygenation using molybdenum isotopes
}

\author{
ZHENG QIN $^{1}$, DONGTAO XU ${ }^{1}$, BRIAN KENDALL ${ }^{2}$, \\ XINGLIANG ZHANG ${ }^{3}$, QIANG OU ${ }^{1}$, XINQIANG WANG ${ }^{1}$, \\ JIE LI ${ }^{4}$ AND JINGAO LIU ${ }^{1}$ \\ ${ }^{1}$ China University of Geosciences (Beijing) \\ ${ }^{2}$ University of Waterloo \\ ${ }^{3}$ Northwest University \\ ${ }^{4}$ Guangzhou Institute of Geochemistry, Chinese Academy of \\ Sciences \\ Presenting Author: qinzheng@cugb.edu.cn
}

Oceanic redox changes have been recognized as a key environmental trigger for the "Cambrian explosion". Globally widespread ocean oxygenation during the early Cambrian has been inferred from high molybdenum (Mo) isotope ratios in sedimentary rocks. However, other redox indicators contradict the Mo isotope data and instead point to the widespread occurrence of bottom water anoxia/euxinia on continental margins at this time. Here, we use new and existing Mo isotope data for black shales deposited at multiple locations on the Yangtze Platform together with a novel isotope mass balance model to re-evaluate the ocean redox structure during Cambrian Age 2 and Age 3. Our results suggest that early Cambrian oceans were not pervasively oxygenated to the same extent as modern oceans, but rather were redox-stratified with euxinic continental margins. Such a redox structure promoted the operation of an $\mathrm{Fe}$ Mn (hydr) oxide shuttle that enhanced delivery of lighter-mass Mo isotopes to sediments and enriched global seawater in heavier-mass Mo isotopes. Modeling reveals that it is possible for the global seawater Mo isotope composition to reach modern values in an early Cambrian ocean that is less oxygenated than the modern ocean. Reconsideration of temporal trends in the Mo isotope composition of sedimentary rocks suggests that time intervals with Mo isotope ratios spanning more than 3\%, including the Ediacaran-Cambrian and Permian-Triassic transitions, were probably affected by Fe-Mn shuttling on euxinic continental margins. Widespread ocean oxygenation like that of the modern ocean may have first occurred in the Late Devonian rather than at the Ediacaran-Cambrian transition. Links between bio-diversification, ecological expansion, and marine redox conditions disclose the importance of redox structures in changing biodiversity evolution. 